\title{
Elevated Red Cell Distribution Width to Platelet Ratio Is Associated With Poor Prognosis in Patients With Spontaneous, Deep-Seated Intracerebral Hemorrhage
}

\section{OPEN ACCESS}

Edited by:

Sean Ruland,

Loyola University Medical Center,

United States

Reviewed by:

Benjamin Aaron Emanuel,

University of Southern California,

United States

Alessandro Biffi,

Massachusetts General Hospital and

Harvard Medical School,

United States

*Correspondence:

Felix Lehmann

felix.lehmann@ukbonn.de

Specialty section:

This article was submitted to

Neurocritical and Neurohospitalist

Care,

a section of the journal

Frontiers in Neurology

Received: 01 August 2021

Accepted: 22 October 2021

Published: 15 November 2021

Citation:

Lehmann F, Schenk LM,

Bernstock JD, Bode C, Borger V

Gessler FA, Güresir E, Hamed M,

Potthoff A-L, Putensen C

Schneider M, Zimmermann J,

Vatter $\mathrm{H}$, Schuss $P$ and

Hadjiathanasiou A (2021) Elevated

Red Cell Distribution Width to Platelet

Ratio Is Associated With Poor

Prognosis in Patients With

Spontaneous, Deep-Seated

Intracerebral Hemorrhage.

Front. Neurol. 12:751510.

doi: 10.3389/fneur.2021.751510
Felix Lehmann ${ }^{1 *}$, Lorena M. Schenk ${ }^{2}$, Joshua D. Bernstock ${ }^{3}$, Christian Bode ${ }^{1}$, Valeri Borger ${ }^{2}$, Florian A. Gessler ${ }^{4}$, Erdem Güresir' ${ }^{2}$, Motaz Hamed ${ }^{2}$, Anna-Laura Potthoff ${ }^{2}$, Christian Putensen ${ }^{1}$, Matthias Schneider ${ }^{2}$, Julian Zimmermann ${ }^{5}$, Hartmut Vatter ${ }^{2}$, Patrick Schuss ${ }^{2}$ and Alexis Hadjiathanasiou ${ }^{2}$

\footnotetext{
${ }^{1}$ Department of Anesthesiology and Intensive Care, University Hospital Bonn, Bonn, Germany, ${ }^{2}$ Department of Neurosurgery, University Hospital Bonn, Bonn, Germany, ${ }^{3}$ Department of Neurosurgery, Brigham and Women's Hospital and Harvard Medical School, Boston, MA, United States, ${ }^{4}$ Department of Neurosurgery, University Hospital Rostock, Rostock, Germany, ${ }^{5}$ Department of Neurology, University Hospital Bonn, Bonn, Germany
}

Object: Inflammatory response is an important determinant of subsequent brain injury after deep-seated intracerebral hemorrhage $(\mathrm{ICH})$. The ratio of red blood cell (RBC) distribution width to platelet count (RPR) has been established as a new index to reflect the severity of inflammation. To the best of our knowledge, no association between RPR and prognosis after spontaneous $\mathrm{ICH}$ has yet been reported.

Methods: In all patients with deep-seated ICH treated at our Neurovascular Center from 2014 to 2020, initial laboratory values were obtained to determine RPR in addition to patient characteristics and known risk factors. Subsequent multivariate analysis was performed to identify independent risk factors for 90-day mortality after deep-seated ICH.

Results: Hundred and two patients with deep-seated ICH were identified and further analyzed. Patients with an initial RPR $<0.06$ exhibited significantly lower mortality rate after 90 days than those with an initial $R P R \geq 0.06$ (27 vs. $57 \% ; p=0.003$ ). Multivariate analysis identified "ICH score $\geq 3$ " ( $p=0.001)$, "anemia on admission" $(p=0.01)$, and "elevated RPR $\geq 0.06$ " ( $p=0.03$ ) as independent predictors of 90-day mortality.

Conclusions: The present study constitutes the first attempt to demonstrate that the ratio of RBC distribution width to platelets - as an independent inflammatory marker-might serve for prognostic assessment in deep-seated $\mathrm{ICH}$.

Keywords: spontaneous intracerebral hemorrhage, red blood cell distribution width, platelets, morbidity/mortality, inflammation

\section{INTRODUCTION}

Patients that suffer from spontaneous intracerebral hemorrhage (ICH) often display significant morbidity/mortality due to the extent and/or the location of the bleed (1). These devastating injuries occur as a result of primary [i.e., mass effect(s)] and/or secondary (i.e., oxidative stress, inflammation, etc.) injury caused by the hemorrhage (2). Of note, 
deep-seated ICH appears to be distinct from lobar ICH, implying different etiologies and ultimately different clinical outcomes (3).

Given the varying degrees and locations of $\mathrm{ICH}$, reliable prognostic aids supporting early clinical decision-making are essential. Accordingly, herein we assessed ICH scores, and a myriad of lab values in effort to develop a tool capable of providing better clinical guidance for neurointensivists, neurosurgeons, ICH patients, and their families (4-7).

Of note, the inflammatory response referenced above is exacerbated via the intracerebral extravasation of blood products $(8,9)$. Intracerebral hemorrhage-induced inflammation precedes disruption of the blood-brain barrier (BBB) and in patients with spontaneous $\mathrm{ICH}$, concurrent alterations of immune profiles within peripheral blood have been observed (10). Such alterations in peripheral blood have been the target of numerous efforts to establish markers for early ICH prognostication (11-13).

Red cell distribution width (RDW), an indicator of size variability among circulating red blood cells (RBCs), is routinely reported as part of the complete blood count (CBC) and is typically used to identify the etiology of anemia (14). More recently, RDW has gained considerable attention as an inflammatory marker and as a predictive metric for cardiac as well as infectious diseases (15-17). In addition, there is evidence that RDW is a significant prognostic factor in several malignancies $(18,19)$. A new index, the ratio of RDW to platelet count (RPR), has been reported to reflect the severity of inflammation and has previously been utilized for prognostic prediction in breast cancer or fibrosis in chronic hepatitis $(20,21)$.

To the best of our knowledge, no association between RPR and prognosis after spontaneous ICH has been reported. Therefore, the aim of the present work was to include the RPR as a simple, readily available index for early prognostic assessment in patients with spontaneous deep-seated ICH to facilitate intensive care decision making.

\section{MATERIALS AND METHODS}

This retrospective study includes 102 patients with spontaneous deep-seated ICH who were referred to our Neurovascular Center for further management between 2014 and July 2020. The institutional ethics review board approved this retrospective study (no. 383/20). All procedures used in this study were performed in accordance with the principles of the Declaration of Helsinki and its subsequent amendments. To obtain a more homogeneous patient cohort, patients with traumatic or other bleeding sources (e.g., aneurysm, arteriovenous malformation, tumor) and those with a lobar ICH location were excluded from further analysis. All patients received at minimum a conservative treatment, including blood pressure control, according to current guidelines. Information on patient characteristics, ICH localization, ICH extent, ICH score (4), and intensive care and laboratory values were collected and analyzed in a computerized database. Furthermore, patients were separated into two groups based on the ICH score. Given the experience from previous studies on the clinical significance of the ICH score pertaining to mortality, the groups were divided into patients with an initial $\mathrm{ICH}$ score $<3$ and an ICH score $\geq 3(4,22)$. Blood samples were obtained from peripheral venous blood as part of routine management at the time of hospital admission and thus before any therapeutic interventions. Red cell distribution width and platelet count were routinely obtained. The ratio of RDW to platelets was calculated accordingly at follow-up. Regarding further inflammatory markers, patients were divided into two groups each based on their C-reactive protein (CRP; CRP $\leq 3$ $\mathrm{mg} / \mathrm{l}$ vs. $\mathrm{CRP}>3 \mathrm{mg} / \mathrm{l})$, procalcitonin value $(\mathrm{PCT}$; $\mathrm{PCT} \leq 0.5$ $\mu \mathrm{g} / \mathrm{l}$ vs. $\mathrm{PCT}>0.05 \mu \mathrm{g} / \mathrm{l})$, and peripheral white blood count (WBC; WBC $\leq 12 \mathrm{~g} / \mathrm{l}$ vs. WBC $>12 \mathrm{~g} / \mathrm{l}$ ) at the time of hospital admission (6). Anemia assessed in the admission laboratory was defined according to the World Health Organization (WHO) definitions [hemoglobin $(\mathrm{Hb})<12 \mathrm{~g} / \mathrm{dl}$ for women, $\mathrm{Hb}<13 \mathrm{~g} / \mathrm{dl}$ for men] (23).

Mortality at 90 days after the bleeding event was used as the primary end point. Thus, to avoid confounding bias of the results attributable to cases with devastating extent of hemorrhage and/or patient wishes of waiving life-sustaining measures, only patients hospitalized for $>3$ days were included. Data analysis was accomplished using the SPSS computer software package (version 25, IBM Corp., Armonk, NY, USA). The Mann-Whitney U-test was chosen to compare continuous variables because the data were mostly not normally distributed. Categorical variables were analyzed in contingency tables using Fisher's exact test. Results with $p<0.05$ were deemed statistically significant. To assess the discrimination ability of the ICH score and the RPR in predicting the probability of a case fatality within 90 days, receiver operating characteristic (ROC) curves were constructed for both values within the patient population studied, and the areas under the curves (AUC) were calculated. The optimal cut-off value for each curve was determined for sensitivity and specificity, both being equally important. In addition, to ascertain independent predictors of 90-day mortality in patients with deepseated ICH, multivariate analysis was performed using binary logistic regression.

\section{RESULTS}

\section{Patient Characteristics}

Between 2014 and July 2020, 102 patients with deep-seated ICH were referred to the corresponding authors' neurovascular specialty center for management/treatment of ICH. The median age of the patients was 66 years [interquartile range (IQR) 57-76 years]. At the time of admission, 64 patients (63\%) presented with a Glasgow Coma Score (GCS) < 13, 63 patients $(62 \%)$ suffered from additional intraventricular hemorrhage (IVH), 53 patients (52\%) had deep-seated ICH with hematoma volume $\geq 30 \mathrm{ml}$. The 90 -day mortality rate was $42 \%$ (43/102 patients). Further details on patient characteristics, focusing on availability of data at the time of admission and stratified by 90 -day mortality into two groups for survivors and non-survivors, are given in Table $\mathbf{1 .}$

\section{Influence of Anemia and Routine Inflammatory Markers on Mortality}

Patients with anemia at the time of admission had a significant difference in 90-day mortality compared to patients without initial anemia (69 vs. $32 \%$; $p=0.001$, OR 4.8 , 95\% CI $1.9-$ 12.2). No significant difference in mortality was observed in 
TABLE 1 | Patient characteristics.

\begin{tabular}{|c|c|c|c|}
\hline & $\begin{array}{l}\text { Survivors } \\
(n=59)\end{array}$ & $\begin{array}{c}\text { Non-survivors } \\
(n=43)\end{array}$ & \\
\hline Median age (years, IQR) & $63(53-72)$ & $68(59-81)$ & $p=0.03$ \\
\hline Female sex & 21 (36\%) & 16 (37\%) & $p=1.0$ \\
\hline $\mathrm{ICH}$ score $>3$ & $4(7 \%)$ & 16 (37\%) & $\begin{array}{l}p=0.0002, \mathrm{OR} \\
8.1,95 \% \mathrm{Cl} \\
2.5-26.7\end{array}$ \\
\hline Initial SBP (mmHg) & & & $p=0.7$ \\
\hline Mild (<180) & $36(61 \%)$ & 27 (63\%) & \\
\hline Moderate (180-219) & 17 (29\%) & $10(23 \%)$ & \\
\hline Severe $(\geq 220)$ & $6(10 \%)$ & $6(14 \%)$ & \\
\hline $\begin{array}{l}\text { Admission CRP } \\
\text { (median, IQR; mg/l) }\end{array}$ & $3.1(1.2-9.2)$ & $3.7(1.5-10.4)$ & $p=0.6$ \\
\hline $\begin{array}{l}\text { Admission PCT (median, } \\
\text { IQR; } \mu \mathrm{g} / \mathrm{l})\end{array}$ & $0.06(0.04-0.12)$ & $0.09(0.05-0.27)$ & $p=0.01$ \\
\hline
\end{tabular}

patients with an initial WBC of $>12 \mathrm{~g} / \mathrm{l}$ compared to patients with a baseline WBC $\leq 12 \mathrm{~g} / \mathrm{l}$ (47 vs. $39 \% ; p=0.5$ ). Ninetyday mortality following ICH was not significant in patients with a baseline CRP $\leq 3 \mathrm{mg} / \mathrm{l}$ compared with patients with an initial CRP $>3 \mathrm{mg} / \mathrm{l}$ (38 vs. $45 \%$; $p=0.6$ ). Patients with deep-seated ICH and an initial PCT $\leq 0.5 \mu \mathrm{g} / \mathrm{l}$ exhibited significantly lower mortality at 90 days compared to patients with an initial PCT $>0.5 \mu \mathrm{g} / \mathrm{l}$ (39 vs. $78 \%$; $p=0.03$, OR 5.5 , 95\% CI 1.1-28.2).

\section{Influence of RPR on Mortality}

Neither the median admission laboratory value for RDW nor that for platelet count demonstrated a significant difference between survivors and non-survivors with deep-seated $\mathrm{ICH}(p=0.2$ and $p=0.07$, respectively). The median RPR for the entire cohort was 0.062 (IQR 0.050-0.078). The optimal cut-off value in terms of RPR was determined to be 0.06 with an AUC of 0.63 (95\% CI $0.517-0.739 ; p=0.027)$ and a sensitivity of $70 \%$ and a specificity of $61 \%$. Table 2 illustrates the distribution of known and established risk factors for poor outcome in patients with deep-seated ICH. With the exception of age, none of the known prognostic factors are statistically different between the two groups. Patients with an RPR $<0.06$ were distinguished by significantly lower case-fatality at 90 days compared to patients with a baseline RPR $\geq 0.06$ (27 vs. $57 \% ; p=0.003$, OR 3.6, 95\% 1.6-8.3).

\section{Multivariate Analysis}

Multivariate logistic regression analysis was performed to identify independent initial determinable predictors of 90-day mortality in patients with deep-seated $\mathrm{ICH}$ and to clarify the influence of the RPR in this regard. Multivariate analysis identified "ICH score $\geq 3$ " ( $p=0.001$, OR 8.6, 95\% CI 2.4-31.1), "anemia on admission" ( $p=0.01$, OR 3.6, 95\% CI 1.3-10.2), and "elevated RPR $\geq 0.06$ " ( $p=0.03$, OR 2.9, 95\% CI 1.1-7.7) as significant and independent predictors of 90-day mortality (Nagelkerke's $R^{2}=0.35$, Figure 1 ).
TABLE 2 | Distribution of known prognostic factors in patients with deep-seated $\mathrm{ICH}$.

\begin{tabular}{lccc}
\hline & $\begin{array}{c}\text { RPR }<\mathbf{0 . 0 6} \\
(\boldsymbol{n}=\mathbf{4 9 )}\end{array}$ & $\begin{array}{c}\text { RPR } \geq \mathbf{0 . 0 6} \\
(\boldsymbol{n}=\mathbf{5 3})\end{array}$ & \\
\hline ICH score $>3$ & $8(16 \%)$ & $12(23 \%)$ & $p=0.5$ \\
Age $\geq 80$ years & $4(8 \%)$ & $13(25 \%)$ & $p=0.03$, OR 3.7, \\
& & & $95 \%$ Cl $1.1-12.1$ \\
Baseline GCS & $30(61 \%)$ & $34(64 \%)$ & $p=0.8$ \\
13 & $22(45 \%)$ & $31(59 \%)$ & $p=0.2$ \\
Baseline ICH & & & $p=0.2$ \\
volume $\geq 30$ ml & $27(55 \%)$ & $36(68 \%)$ & $p=0.1$ \\
Initial IVH & $2(4 \%)$ & $8(15 \%)$ & $p=0.7$ \\
CRRT & $21(43 \%)$ & $25(47 \%)$ & $p=0.003$, OR \\
PMV $(>7$ days) & $13(27 \%)$ & $30(57 \%)$ & $3.6,95 \% 1.6-8.3$ \\
90 day mortality & & &
\end{tabular}

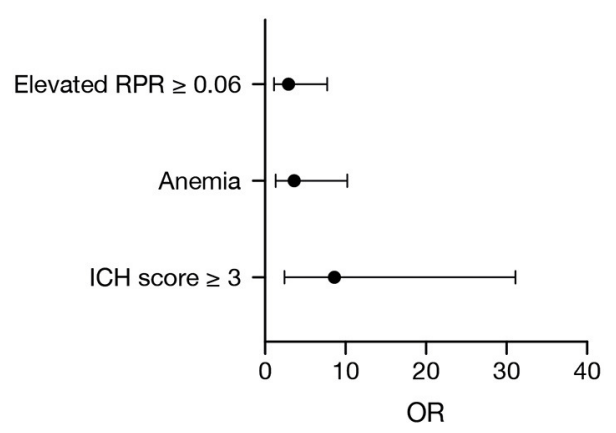

FIGURE 1 | Forest plot of significant and independent predictors of 90-day mortality identified by multivariate logistic regression analysis.

\section{DISCUSSION}

The present study evaluated a potential link between RPR and 90-day mortality after deep-seated ICH. The results suggest that patients with an elevated RPR in the admission laboratory are more likely to decease within 90 days after the bleeding.

RPR is calculated from the ratio of RBC distribution width to platelet count. As a potential indicator of inflammatory processes, RPR has already been established as a prognostic factor in numerous other diseases. In patients with $\mathrm{ICH}$, to the best of our knowledge, there have been no such association studies yet. In the context of the initial bleeding, but also in the course of the alteration/depletion processes in the area of the hemorrhage, a number of inflammatory reactions have been detected in ICH $(24,25)$. Not only via the initial extravasation of cytokines, but also through blood breakdown products, inflammatory reactions may very well have a direct impact on the pathophysiological and clinical course, e.g., on the development of perilesional edema (26). Thus, early and readily available inflammatory markers may provide an important insight into the inflammatory events early in the course of management/treatment of patients with ICH and thereby offer 
a reflection of the inflammation-triggered brain injury in the setting of ICH $(27,28)$.

RPR is derived in part from RDW. Red cell distribution width measures the size distribution of RBCs and indicates in case of strongly elevated values the presence of different types of anemia. Anemia or low hemoglobin have already been identified as influencing factors on outcome but also on hematoma extent after spontaneous ICH (29-31). In the present work, anemia is also associated with increased mortality, and yet the RPR extends beyond a mere determination of hemoglobin by including a platelet count. RPR has been found to be a prognostic marker in various immunological diseases and is also considered to reflect the severity of the inflammatory event by attributing some influence to platelet count $(20,21)$. In the inflammatory process, in addition to sepsis-related anemic events, inflammatory cytokines may inhibit the maturation of RBCs and thus alter the RDW (32) whereas platelets are known to be placed on the intersection of an immune response and coagulation in infectious diseases (33). Thus, a marker as RPR, which takes into account both potential reflections of the inflammatory process, may be more accurate than nonspecific markers such as CRP $(34,35)$. Also, in the anticipation of further complications in the course of treatment of ICH (e.g., renal replacement therapy), non-specific markers such as CRP have been reported to be less predictive than those that are considered more specific for inflammatory processes, such as PCT (6). Common to non-specific inflammatory markers may be a reflection of just another surrogate parameter as for example, in terms of a stress response of the body to intracranial hematoma volume or aspiration in the context of low GCS.

Nevertheless, when discussing the potential implications of this readily available laboratory marker, it is important to note that the focus of the present study was directed at patients with deep-seated ICH. One reason for this selection lies in the multiple etiologic possibilities in patients with lobar ICH. An underlying disease resulting in lobar ICH (e.g., cancer) seems to be more likely to affect initial laboratory parameters compared to deep-seated ICH (3).

The present study demonstrates the anticipated distribution of known and/or established risk factors for increased mortality after ICH (except for age) in both groups with decreased and increased RPR. This strengthens the suspicion that the RPR could be established as an independent prognostic factor. Despite the association of RPR with mortality after deep-seated ICH reported herein, further exploration of the underlying mechanisms and corroboration of our findings in other study populations is warranted.

\section{REFERENCES}

1. Hemphill JC III, Greenberg SM, Anderson CS, Becker K, Bendok BR, Cushman $\mathrm{M}$, et al. Guidelines for the management of spontaneous intracerebral hemorrhage: a guideline for healthcare professionals from the American Heart Association/American Stroke Association. Stroke. (2015) 46:2032-60. doi: 10.1161/STR.0000000000000069

\section{LIMITATIONS}

In addition to retrospective data collection and analysis with the bias inherent to this study design, our study reveals other shortcomings that need to be carefully considered when interpreting our results. The sample size is comparably small due to a restriction to patients with a deep-seated ICH. This increases the homogeneity of the study population, but also raises the possibility of selection bias. Among these is the exclusion of patients with lobar ICH. However, this might also be considered a potential advantage, as this pre-selection might reduce the variety of underlying ICH etiologies and therefore their potential influence on initial laboratory values. In addition, the retrospective data analysis also precludes a dedicated analysis of potential additional determinants (such as a preexisting chronic inflammatory disease/acute aspiration pneumonia) throughout the complete dataset.

\section{CONCLUSION}

This is the first study to provide evidence that RBC distribution width to platelet ratio might constitute an independent marker of inflammation associated with the prognostication of outcome in deep-seated ICH.

\section{DATA AVAILABILITY STATEMENT}

The original contributions presented in the study are included in the article/supplementary material, further inquiries can be directed to the corresponding author/s.

\section{ETHICS STATEMENT}

The studies involving human participants were reviewed and approved by Local Ethics Committee at the University of Bonn. Written informed consent for participation was not required for this study in accordance with the national legislation and the institutional requirements.

\section{AUTHOR CONTRIBUTIONS}

FL, PS, and AH: conceptualization and supervision. FL, LS, $\mathrm{PS}$, and $\mathrm{AH}$ : data curation. $\mathrm{FL}, \mathrm{PS}, \mathrm{MS}$, and $\mathrm{AH}$ : formal analysis. FL, JB, FG, MS, PS, and AH: writing-original draft preparation. FL, LS, JB, CB, VB, FG, EG, MH, A-LP, CP, MS, JZ, HV, PS, and AH: writing-review and editing. A-LP and MS: visualization. All authors contributed to the article and approved the submitted version.

2. Zhu H, Wang Z, Yu J, Yang X, He F, Liu Z, et al. Role and mechanisms of cytokines in the secondary brain injury after intracerebral hemorrhage. Prog Neurobiol. (2019) 178:101610. doi: 10.1016/j.pneurobio.2019. 03.003

3. Schuss P, Bode C, Borger V, Coch C, Guresir A, Hadjiathanasiou A, et al. MR-imaging and histopathological diagnostic work-up of patients with spontaneous lobar intracerebral hemorrhage: results 
of an institutional prospective registry study. Diagnostics. (2021) 11:368. doi: 10.3390/diagnostics11020368

4. Hemphill JC III, Bonovich DC, Besmertis L, Manley GT, Johnston SC. The ICH score: a simple, reliable grading scale for intracerebral hemorrhage. Stroke. (2001) 32:891-7. doi: 10.1161/01.str.32.4.891

5. Hwang DY, Chu SY, Dell CA, Sparks MJ, Watson TD, Langefeld $\mathrm{CD}$, et al. Factors considered by clinicians when prognosticating intracerebral hemorrhage outcomes. Neurocrit Care. (2017) 27:316-25. doi: 10.1007/s12028-017-0430-7

6. Schenk LM, Schneider M, Bode C, Guresir E, Junghanns C, Muller M, et al. Early laboratory predictors for necessity of renal replacement therapy in patients with spontaneous deep-seated intracerebral hemorrhage. Front Neurol. (2021) 12:636711. doi: 10.3389/fneur.2021.636711

7. Tu L, Liu X, Li T, Yang X, Ren Y, Zhang Q, et al. Admission serum calcium level as a prognostic marker for intracerebral hemorrhage. Neurocrit Care. (2019) 30:81-7. doi: 10.1007/s12028-018-0574-0

8. Zhou Y, Wang Y, Wang J, Anne Stetler R, Yang QW. Inflammation in intracerebral hemorrhage: from mechanisms to clinical translation. Prog Neurobiol. (2014) 115:25-44. doi: 10.1016/j.pneurobio.2013.11.003

9. Lan X, Han X, Liu X, Wang J. Inflammatory responses after intracerebral hemorrhage: from cellular function to therapeutic targets. J Cereb Blood Flow Metab. (2019) 39:184-6. doi: 10.1177/0271678X18805675

10. Lattanzi S, Brigo F, Trinka E, Cagnetti C, Di Napoli M, Silvestrini M. Neutrophil-to-lymphocyte ratio in acute cerebral hemorrhage: a system review. Transl Stroke Res. (2019) 10:137-45. doi: 10.1007/s12975-018-0649-4

11. Walsh KB, Sekar P, Langefeld CD, Moomaw CJ, Elkind MS, Boehme AK, et al. Monocyte count and 30-day case fatality in intracerebral hemorrhage. Stroke. (2015) 46:2302-4. doi: 10.1161/STROKEAHA.115.009880

12. Saand AR Yu F, Chen J, Chou SH. Systemic inflammation in hemorrhagic strokes - a novel neurological sign and therapeutic target? J Cereb Blood Flow Metab. (2019) 39:959-88. doi: 10.1177/0271678X19841443

13. Agnihotri S, Czap A, Staff I, Fortunato G, McCullough LD. Peripheral leukocyte counts and outcomes after intracerebral hemorrhage. $J$ Neuroinflammation. (2011) 8:160. doi: 10.1186/1742-2094-8-160

14. Salvagno GL, Sanchis-Gomar F, Picanza A, Lippi G. Red blood cell distribution width: a simple parameter with multiple clinical applications. Crit Rev Clin Lab Sci. (2015) 52:86-105. doi: 10.3109/10408363.2014.992064

15. Aung N, Dworakowski R, Byrne J, Alcock E, Deshpande R, Rajagopal K, et al. Progressive rise in red cell distribution width is associated with poor outcome after transcatheter aortic valve implantation. Heart. (2013) 99:12616. doi: 10.1136/heartjnl-2013-303910

16. Foy BH, Carlson JCT, Reinertsen E, Padros IVR, Pallares Lopez R, PalanquesTost E, et al. Association of red blood cell distribution width with mortality risk in hospitalized adults with SARS-CoV-2 infection. JAMA Network Open. (2020) 3:e2022058. doi: 10.1001/jamanetworkopen.2020. 22058

17. Uffen JW, Oomen P, de Regt M, Oosterheert JJ, Kaasjager K. The prognostic value of red blood cell distribution width in patients with suspected infection in the emergency department. BMC Emerg Med. (2019) 19:76. doi: 10.1186/s12873-019-0293-7

18. Kaisman-Elbaz T, Elbaz Y, Merkin V, Dym L, Noy A, Atar-Vardi M, et al. Hemoglobin levels and red blood cells distribution width highlights glioblastoma patients subgroup with improved median overall survival. Front Oncol. (2020) 10:432. doi: 10.3389/fonc.2020.00432

19. Wang PF, Song SY, Guo H, Wang TJ, Liu N, Yan CX. Prognostic role of pretreatment red blood cell distribution width in patients with cancer: a metaanalysis of 49 studies. J Cancer. (2019) 10:4305-17. doi: 10.7150/jca.31598

20. Takeuchi H, Abe M, Takumi Y, Hashimoto T, Miyawaki M, Okamoto $\mathrm{T}$, et al. Elevated red cell distribution width to platelet count ratio predicts poor prognosis in patients with breast cancer. Sci Rep. (2019) 9:3033. doi: 10.1038/s41598-019-40024-8

21. Taefi A, Huang CC, Kolli K, Ebrahimi S, Patel M. Red cell distribution width to platelet ratio, a useful indicator of liver fibrosis in chronic hepatitis patients. Hepatol Int. (2015) 9:454-60. doi: 10.1007/s12072-015-9638-9
22. Lehmann F, Schenk LM, Ilic I, Putensen C, Hadjiathanasiou A, Borger $\mathrm{V}$, et al. Prolonged mechanical ventilation in patients with deep-seated intracerebral hemorrhage: risk factors and clinical implications. J Clin Med. (2021) 10:1015. doi: $10.3390 / \mathrm{jcm} 10051015$

23. Nutritional anaemias. Report of a WHO scientific group. World Health Organ Tech Rep Ser. (1968) 405:5-37.

24. Wang J, Dore S. Inflammation after intracerebral hemorrhage. J Cereb Blood Flow Metab. (2007) 27:894-908. doi: 10.1038/sj.jcbfm.9600403

25. Tschoe C, Bushnell CD, Duncan PW, Alexander-Miller MA, Wolfe SQ. Neuroinflammation after intracerebral hemorrhage and potential therapeutic targets. J Stroke. (2020) 22:29-46. doi: 10.5853/jos.2019.02236

26. Ironside N, Chen CJ, Ding D, Mayer SA, Connolly ES Jr. Perihematomal edema after spontaneous intracerebral hemorrhage. Stroke. (2019) 50:162633. doi: 10.1161/STROKEAHA.119.024965

27. Chen S, Yang Q, Chen G, Zhang JH. An update on inflammation in the acute phase of intracerebral hemorrhage. Transl Stroke Res. (2015) 6:48. doi: 10.1007/s12975-014-0384-4

28. Ren H, Han R, Chen X, Liu X, Wan J, Wang L, et al. Potential therapeutic targets for intracerebral hemorrhage-associated inflammation: an update. $J$ Cereb Blood Flow Metab. (2020) 40:1752-68. doi: 10.1177/0271678X20923551

29. Kumar MA, Rost NS, Snider RW, Chanderraj R, Greenberg SM, Smith EE, et al. Anemia and hematoma volume in acute intracerebral hemorrhage. Crit Care Med. (2009) 37:1442-7. doi: 10.1097/CCM.0b013e31819ced3a

30. Roh DJ, Albers DJ, Magid-Bernstein J, Doyle K, Hod E, Eisenberger A, et al. Low hemoglobin and hematoma expansion after intracerebral hemorrhage. Neurology. (2019) 93:e372-80. doi: 10.1212/WNL.0000000000007820

31. Kuramatsu JB, Gerner ST, Lucking H, Kloska SP, Schellinger PD, Kohrmann $\mathrm{M}$, et al. Anemia is an independent prognostic factor in intracerebral hemorrhage: an observational cohort study. Crit Care. (2013) 17:R148. doi: 10.1186/cc12827

32. Pierce CN, Larson DF. Inflammatory cytokine inhibition of erythropoiesis in patients implanted with a mechanical circulatory assist device. Perfusion. (2005) 20:83-90. doi: 10.1191/0267659105pf793oa

33. Manne BK, Xiang SC, Rondina MT. Platelet secretion in inflammatory and infectious diseases. Platelets. (2017) 28:15564. doi: 10.1080/09537104.2016.1240766

34. Ge S, Lin S, Zhang L, Zeng M. The association of red blood cell distribution width to platelet count ratio and 28-day mortality of patients with sepsis: a retrospective cohort study. Ther Clin Risk Manag. (2020) 16:999-1006. doi: 10.2147/TCRM.S268523

35. Lopponen P, Qian C, Tetri S, Juvela S, Huhtakangas J, Bode MK, et al. Predictive value of C-reactive protein for the outcome after primary intracerebral hemorrhage. J Neurosurg. (2014) 121:1374-9. doi: 10.3171/2014.7.JNS132678

Conflict of Interest: The authors declare that the research was conducted in the absence of any commercial or financial relationships that could be construed as a potential conflict of interest.

Publisher's Note: All claims expressed in this article are solely those of the authors and do not necessarily represent those of their affiliated organizations, or those of the publisher, the editors and the reviewers. Any product that may be evaluated in this article, or claim that may be made by its manufacturer, is not guaranteed or endorsed by the publisher.

Copyright (C) 2021 Lehmann, Schenk, Bernstock, Bode, Borger, Gessler, Güresir, Hamed, Potthoff, Putensen, Schneider, Zimmermann, Vatter, Schuss and Hadjiathanasiou. This is an open-access article distributed under the terms of the Creative Commons Attribution License (CC BY). The use, distribution or reproduction in other forums is permitted, provided the original author(s) and the copyright owner(s) are credited and that the original publication in this journal is cited, in accordance with accepted academic practice. No use, distribution or reproduction is permitted which does not comply with these terms. 